\title{
Expression of the adaptor protein Tks5 in human cancer: Prognostic potential
}

\author{
STANLEY S. STYLLI ${ }^{1,2}$, RODNEY B. LUWOR ${ }^{1}$, ANDREW H. KAYE ${ }^{1,2}$, \\ STACEY T.T.I ${ }^{3}$, CHRISTOPHER M. HOVENS ${ }^{2}$ and PETER LOCK ${ }^{3}$
}

\author{
${ }^{1}$ Department of Surgery, The University of Melbourne, The Royal Melbourne Hospital, Parkville, Victoria 3052; \\ ${ }^{2}$ Department of Neurosurgery, The Royal Melbourne Hospital, Parkville, Victoria 3050; ${ }^{3}$ Department of Biochemistry, \\ La Trobe Institute of Molecular Sciences, La Trobe University, Bundoora, Victoria 3086, Australia
}

Received April 21, 2014; Accepted June 4, 2014

DOI: $10.3892 / o r .2014 .3310$

\begin{abstract}
Tks5 (tyrosine kinase substrate with $5 \mathrm{SH} 3$ domains) is an adaptor protein which cooperates with Src tyrosine kinase to promote the formation of protease-enriched, actin-based projections known as invadopodia, which are utilized by invasive cancer cells to degrade the extracellular matrix (ECM). We previously identified a Src-Tks5-Nck pathway which promotes invadopodium formation and ECM proteolysis in melanoma and breast cancer cells. We therefore sought to investigate the significance of Tks5 expression in human cancers. This was undertaken retrospectively through an immunohistochemical evaluation in tissue microarray cores and through data mining of the public database, Oncomine. Here we showed that Tks5 was expressed at higher levels in the microarray cores of breast, colon, lung and prostate cancer tissues compared to the levels in normal tissues. Importantly, mining of Oncomine datasets revealed a strong correlation between Tks 5 mRNA overexpression in a number of cancers with increased metastatic events and a poorer prognosis. Collectively, these findings suggest a clinical association of Tks5 expression in human cancers. It identifies the importance for further investigations in examining the full potential of Tks5 as a relevant prognostic marker in a select number of cancers which may have implications for future targeted therapies.
\end{abstract}

Correspondence to: Dr Stanley S. Stylli, Department of Surgery, The University of Melbourne, The Royal Melbourne Hospital, Parkville, Victoria 3052, Australia

E-mail: stanley.stylli@mh.org.au

Abbreviations: DMEM, Dulbecco's modified Eagle's medium; ECM, extracellular matrix; EGF, epidermal growth factor; EGFR, epidermal growth factor receptor; FCS, foetal calf serum; HRP, horseradish peroxidase; $\mathrm{kDa}$, kilodalton; MMP, matrix metalloproteinase; nM, nanomolar; rpm, revolutions per minute; SDS, sodium dodecyl sulphate; SEM, standard error of the mean; Tks5, tyrosine kinase substrate with 5 SH3 domains; TMA, tissue microarray; WIP, WASP-interacting protein

Key words: Tks5, invadopodia, cancer, prognosis, Oncomine

\section{Introduction}

The cause of death for $~ 90 \%$ of cancer patients is the metastatic spread of cancer cells from the primary tumour and the subsequent development of secondary tumours within heterologous tissues and organs (1). Patients presenting with symptoms related to localized primary disease subsequently receive a range of treatments to eradicate the tumour including surgery, radiation and chemotherapy. Yet, numerous patients return post-therapy with a developed metastatic lesion at a secondary site. This suggests that the dissemination of tumours involving the migration and penetration of tissue barriers by metastatic cells probably occurs prior to a patient presenting with the primary tumour. Tissue invasion and metastasis is regarded as one of the key 'acquired capabilities or hallmarks' of malignant cancer cells proposed by Hanahan and Weinberg (2), and research into the underlying mechanisms of invasion and metastasis has been a major focus in biology.

Many tumour cells with high invasive or metastatic potential possess the ability to form structures known as invadopodia, via the involvement of numerous adaptor, signalling, adhesion and proteolytic proteins (3-14). The proteolytic function of invadopodia is reliant on the cooperative interactions of the underlying network of these proteins and their environment. Therefore, as the role of invadopodia in cancer invasion and metastasis continues to grow, so does the justification for targeting the regulators of invadopodia in the treatment of malignant cancers.

A number of studies have shown that the actin filamentbinding protein cortactin (which is encoded by the EMS1 oncogene on chromosome 11q13) promotes actin polymerization and branching in invadopodium formation $(7,15)$. It is a key regulatory component of invadopodia and has been shown to be overexpressed in a number of breast carcinomas and head and neck cancers that are correlated with tumour invasiveness and poor prognosis (16). Overexpression of cortactin in breast carcinoma cells also results in an increased number of metastases to bone which is regulated by three phosphorylation sites in cortactin (17). Another actin-associated protein, WIP (WASP-interacting protein) shown to be essential for invadopodium formation (18), has also been observed to have a potential role in cancer cell invasion and metastasis (19). 
Analyses were carried out on microarray data sets concerning the expression of WIPF1 as a prognostic signature in colorectal breast cancer and glioma patients with a strong correlation observed between low WIP expression and improved prognosis in these patients. This identifies the evolving relevance of invadopodia in tumour cell dissemination and subsequent cancer mortality.

The epidermal growth factor receptor (EGFR) has also been linked to invadopodium induction as epidermal growth factor (EGF) stimulation was found to boost invadopodium formation and subsequent matrix degradation in mammary carcinoma cells, whereas suppression of receptor activity with the specific inhibitor, AG1478, abolished invadopodium formation (18). N-WASP, a protein linked to Src signalling and invadopodium formation, through its interaction with WIP was also observed to be critical to the establishment of invadopodia through this pathway. The involvement of EGF as one of the drivers of invadopodia is highly significant as EGFR overexpression and mutations occur in many types of cancers (20-22). Tks5 (tyrosine kinase substrate with 5 SH3 domains) is a scaffold protein and a substrate of the tyrosine kinase Src (23), which has been shown to be required for the organization of invadopodia in tumour cells (24). A study by Fekete et al demonstrated that Tks5 is a novel component of the EGF signalling pathway as EGF treatment of cells results in a sustained level of Tks5 phosphorylation and translocation to the plasma membrane (25).

The Tks5 adaptor protein has been shown to be critical for invadopodium formation and function $(3,24,26-28)$. We previously observed that the Src-mediated phosphorylation of Tks5 at tyrosine Y557 promotes its association with the SH3-SH2 domain proteins, Nck1 and Nck2, which in turn drives local actin dynamics and promotes invadopodium formation in B16F10 melanoma cells (24). Recently, an essential role for a Src-Tks5 signalling pathway involving Tks5 tyrosine phosphorylation was also uncovered in the migration of neural crest-derived cells during zebrafish embryogenesis supporting the notion that developmental cell migration might provide default mechanisms that may also drive invasion and metastasis (29).

Tks5 expression has been previously detected in paraffinembedded human specimens of breast cancer and melanoma tissues using only a qualitative immunohistochemical approach (28). In addition, we demonstrated a prognostic relevance for Tks5 expression in a pilot study involving glial-derived brain tumours (30). This emerging role of Tks5 as a possible prognostic marker in cancer led us to undertake a quantitative analysis of Tks5 expression in tissue microarray (TMA) cores of breast, colon, lung and prostate tumour and normal tissues in the present study. We also carried out an extensive mRNA analysis using the Oncomine cancer gene microarray databases to further provide evidence that Tks5 is selectively upregulated in a number of human cancers and that this expression is highly important both in the metastatic potential of tumours and ultimately, in patient survival.

\section{Materials and methods}

Cell line and culture. Cell culture media and the supplements penicillin/streptomycin and L-glutamine were purchased from Gibco (Invitrogen, USA). Foetal calf serum (FCS) was purchased from Bovogen Biologicals (Australia). The matrix metalloproteinase (MMP) inhibitor GM6001 was obtained from Merck Millipore (USA). The following antibodies: p-Src (Tyr416; CST2101) and GAPDH (14C10; CST2118) were from Cell Signalling Technology (USA), while Tks5 (FISH; M-300; sc-30122) and MMP-2 (H-76; sc-10736) were from Santa Cruz Biotechnology (USA) and $\beta$-tubulin (G7121) was from Promega (USA). All general reagents were purchased from Sigma-Aldrich (USA) or Invitrogen (USA). The murine melanoma B16F10 cell line was a gift from Professor David Ashley (Royal Children's Hospital, Parkville, Australia). The cell line was maintained in Dulbecco's modified Eagle's medium (DMEM) supplemented with FCS $(10 \% \mathrm{v} / \mathrm{v})$, penicillin (100 U/ml), streptomycin $(100 \mu \mathrm{g} / \mathrm{ml})$ and $2 \mathrm{mM}$ L-glutamine. It was maintained at $37^{\circ} \mathrm{C}$ with $10 \% \mathrm{CO}_{2}$.

B16F10 stable cell line generation. The $\mathrm{pBI}$ expression vector which contains two (bi-directional) CMV promoters under the control of a tetracycline (doxycycline) responsive element (TRE) was chosen to generate the panel of B16F10 doxycycline inducible cell lines. B16F10 cells were co-transfected with $\mathrm{pBI}$ constructs along with a plasmid encoding the tetracycline repressor under the control of a constitutive promoter and also containing the puromycin resistance gene to enable selection. Under uninduced conditions, the tet repressor protein remains bound to the TRE and represses transcription of the gene of interest. Addition of doxycycline to cells results in binding of doxycycline to the tet repressor and its dissociation from the TRE. This in turn activates transcription of the gene of interest. DNA constructs encoding full length Tks5 or Src were generated by PCR as previously described (23) and inserted into the pBI Bidirectional Tet vector (Clontech, USA) which was kindly provided by Zhu et al (31). B16F10 cells were transfected with a total of $1 \mu \mathrm{g}$ DNA using Effectene (Qiagen, Australia) and stable transfectants were selected using $5 \mu \mathrm{g} / \mathrm{ml}$ puromycin for up to 4 weeks.

SDS page and immunoblot analysis. The B16F10 cells were lysed with lysis buffer and clarified by centrifugation $\left(13,000 \times \mathrm{g}\right.$ for $25 \mathrm{~min}$ at $\left.4^{\circ} \mathrm{C}\right)$. Whole cell lysate $(20 \mu \mathrm{g})$ was separated by SDS-PAGE on NuPAGE 4-12\% Bis-Tris pre-cast gels (Invitrogen), transferred onto PVDF membranes (Millipore). For detection of the antigen, the membranes were incubated with the indicated antibodies on an orbital shaker for $1-2 \mathrm{~h}$ at room temperature or overnight at $4^{\circ} \mathrm{C}$. The signal was then visualized using the ECL chemilluminescence detection kit (GE Healthcare, Australia) following incubation with appropriate secondary HRP antibodies.

Gelatin zymography. B16F10 cells were assayed for the presence of MMP-2 and MMP-9 by gelatin zymography using $10 \%$ gelatin-substrate zymography NuPAGE pre-cast gels (Invitrogen, Australia). Briefly, $20 \mu \mathrm{l}$ of non-heated serum-free culture medium samples was mixed with Novex ${ }^{\circledR}$ Tris-Glycine SDS sample buffer (Invitrogen) and electrophoresed at $125 \mathrm{~V}$ for $90 \mathrm{~min}$. The gels were subsequently renaturated and developed before being stained with Coomassie blue (Novex) to reveal the positions of active gelatinases (clear bands) against the undigested stained 
gelatin substrate in the gel. The molecular size of the gelatinases was determined using molecular weight markers (Bio-Rad).

Matrigel invasion assay. The calcein AM assay and the BD BioCoat $^{\mathrm{TM}}$ Tumor Invasion System (Becton-Dickinson) were utilized to quantify the ability of the pooled B16F10 inducible cell lines to invade through a Matrigel matrix. All cell lines were induced with doxycycline hyclate $(2 \mu \mathrm{g} / \mathrm{ml})$ overnight prior to transfection with the control or Tks5 siRNA duplexes (Santa Cruz). The siRNA is a pool of proprietary 3 targetspecific 19-25 nucleotide siRNAs that were transfected with the non-liposomal lipid reagent, Effectene, as directed by the manufacturer. Forty-eight hours post transfection, cells were trypsinized, the cell suspension density was determined and $5 \times 10^{4}$ cells were seeded per well within the inner chamber in serum-free DMEM in a volume of $500 \mu \mathrm{l}$ with or without $10 \mu \mathrm{M}$ GM6001. A $750 \mu \mathrm{l}$ volume of DMEM with $10 \%$ FCS was added to the bottom chamber via the sample port as a chemoattractant for the cells. The cells were then incubated at $37^{\circ} \mathrm{C}$, in $10 \% \mathrm{CO}_{2}$ for $24 \mathrm{~h}$. The medium was then removed from the inner chamber, and the inserts were transferred to a FluoroBlok 24-well plate containing $500 \mu \mathrm{l} /$ well of $4 \mu \mathrm{g} / \mathrm{ml}$ calcein AM and incubated at $37^{\circ} \mathrm{C}$ for $1 \mathrm{~h}$ as per the manufacturer's protocol. The levels of fluorescent calcein were then measured fluorimetrically at $520 \mathrm{~nm}$.

Preparation of pooled B16F10 inducible cell block. Aliquots of the pooled B16F10 inducible clones were thawed and left in culture medium for $24 \mathrm{~h}$ before being induced with doxycycline $(2 \mu \mathrm{g} / \mathrm{ml})$ overnight. The cells were then trypsinized and gently pelleted at $1,200 \mathrm{rpm}$. The centrifuge tube was then cut open and the pellet carefully transferred with a flat blade spatula to a mould of 2\% low gelling agarose (Sigma Aldrich) that was previously cooled to $37^{\circ} \mathrm{C}$. The blocks were fixed in neutral buffered formalin overnight and then embedded in paraffin. Sections $(5 \mu \mathrm{m})$ were cut and mounted on SuperFrost Plus slides (Menzel).

Antigen retrieval procedure - Tks5 immunohistochemistry. The antigen retrieval procedure outlined below was utilized for the pooled B16F10 inducible cell block sections (as control specimens) and the human cancer tissue microarray (Chemicon Select Tissue Array, TMA2004; Millipore). The TMA2004 microarray consists of 2-mm cores with cancer and normal tissues from breast, colon, lung and prostate cancer patients. The slides were placed in a $60^{\circ} \mathrm{C}$ oven for $1 \mathrm{~h}$ prior to dewaxing and subsequent rehydration. Antigen retrieval was then carried out in a Biocare Decloaking Chamber Plus (Biocare) for $10 \mathrm{~min}$ in a citrate buffer ( $\mathrm{pH}$ 6.0) (Invitrogen). The slides were washed and quenched for endogenous peroxidase activity. Following incubation in a blocking buffer (Vectastain Elite ABC kit; Vector Laboratories), they were then incubated with a Tks5 antibody [1:50 dilution; Santa Cruz, FISH (M-20)] at $4^{\circ} \mathrm{C}$ overnight. Primary antibodies were detected using a biotinylated secondary antibody (Vectastain Elite ABC kit) and developed with the DAB peroxidase substrate kit (both from Vector Laboratories). The slides were then counterstained with haematoxylin before being mounted with DPX (SigmaAldrich).
Image acquisition and evaluation of Tks5 expression. Brightfield images were captured on a Leica DM750 microscope coupled to a Leica ICC50HD camera (Leica Microsystems) with a 10 and $40 \mathrm{X}$ objective using the Leica Application Suite software (version 3.8.0). The tissue microarray slides were reviewed and scored independently by two pathologists blinded to the information supplied with the slides. The final score (total IHC score) was determined by combining the proportion of positively stained cells and the intensity of staining (Total IHC score = tumour cell proportion $\mathrm{x}$ staining intensity). Tumour cell proportion was scored as follows: 1 ( $\leq 25 \%$ positive cells); 2 (26-50\% positive cells); 3 ( $\geq 50 \%$ positive cells). Staining intensity was graded according to the following criteria: 0 (no staining); 1 (weak staining); 2 (moderate staining) and 3 (strong staining). Using this method of assessment, we evaluated the levels of Tks5 expression in the tissue microarray cores.

Oncomine data mining. Oncomine 4.4.4.3 (www.oncomine. org, Compendia Bioscience ${ }^{\mathrm{TM}}$, Ann Arbor, MI, USA, part of Life Technologies) was utilized for the analysis of Tks5 gene expression. Oncomine is an online tool that contains 715 mRNA and copy number expression datasets from 86,733 cancer and normal tissue samples. These datasets are compiled from publicly available cancer microarray data which is then processed using the same criteria before being made available (31). We used the Oncomine compendium to perform differential expression analyses on Tks 5 mRNA expression levels in cancer tissues versus their normal tissue counterparts. A total of 135 datasets examining mRNA expression differences were listed in the compendium comparing the two tissue types, which is subjected to t-statistics with false discovery rates as a corrected measure of significance (32). We then exposed each study dataset to threshold criteria for inclusion in the Tks 5 mRNA expression analysis. The threshold criteria that were initially utilized for this study were a p-value $<0.05$ and an mRNA expression fold-change $>2$. The fold-change is classified as a change in the mRNA expression level in the cancer tissue compared to the normal expression level for that tissue specifically for your gene of interest. Based on the threshold criteria, Oncomine will then assign a gene rank percentile for all genes studied within a dataset. This figure is the percentage ranking of your gene of interest based on the $\mathrm{p}$-value relative to the $\mathrm{p}$-values of all the other genes within the same dataset. However, datasets with fold-changes $<2$ were also further examined as significant differences as designated by the p-values in Tks5 expression were observed. Initially Tks5 expression was examined across a range of cancer types before undertaking analyses examining the relationship between Tks5 expression, survival, time to metastatic events and expression differences between primary and metastatic tissues. The gene expression data generated through Oncomine is log transformed and standard deviation normalized to one per array studied.

Statistical analysis. All of the data were analyzed with GraphPad Prism (GraphPad Software, Inc., La Jolla, CA, USA). Results are expressed as means \pm SEM. Comparisons between two groups were assessed by Student's t-test. Differences between groups were considered significant at 
$\mathrm{p}<0.05$. All experiments were performed at least twice to confirm reproducibility.

\section{Results}

Doxycycline-induced Tks5 overexpression in B16F10 cells. We previously showed a functional link between Src and Tks5 cooperation in the invadopodial regulation of ECM degradation (24). Several MMPs and serine proteinases have been found in association with invadopodia and are thought to contribute to the ECM proteolytic activities of invasive tumour cells including the membrane tethered MT1-MMP and the secreted gelatinases MMP-2 and MMP-9 $(5,6,11,14,33,34)$. In order to investigate the consequences of Tks5 and Src on MMP activity, a panel of B16F10 cell lines was generated to inducibly overexpress Tks5 alone, Src alone or Src and Tks5 in combination. It was envisaged that stable inducible cells would provide a more valid and powerful tool for examining the effects of Tks5 than transiently overexpressing cells. In particular, i) all cells within a population should express the protein of interest and thereby enable relatively small differences between populations of cells to be detected, ii) inducible expression would provide a means of maintaining overexpression for longer periods amenable to long term assays and iii) the inducible system also provides for a negative control group (uninduced cells) that are of the same genotype as the treated cells. Between 20-25 clones corresponding to each group, B16F10 control (empty vector control), B16F10-Src, $\mathrm{B} 16 \mathrm{~F} 10-\mathrm{Tks} 5$ and $\mathrm{B} 16 \mathrm{~F} 10-\mathrm{Src} / \mathrm{Tk} 5$ were selected in puromycin. The cells were then cultured in the presence or absence of doxycycline and further screened for the induction of Src or Tks5 expression (data not shown). Ten independent clones that stably expressed similar levels of Src or Tks5 in response to doxycycline treatment were selected and re-analyzed. They were then pooled in equal numbers in order to minimize clonal variation effects. Doxycycline induced Src or Tks5 expression in the pooled cells is shown in Fig. 1A. Timecourse experiments were performed to determine whether $\mathrm{Src}$ or Tks5 expression could be maintained at elevated levels for extended periods in response to doxycycline (Fig. 1B). Cells were treated with doxycycline for the indicated times and as determined by immunoblot analysis, Tks5 expression was induced at $24 \mathrm{~h}$ and maintained at similar levels for the $96 \mathrm{~h}$ duration of the experiment.

Induction of Src/Tks5 overexpression in B16F10 pooled inducibles enhances MMP-2 secretion and activation. To further address the consequences of Src and Tks5 overexpression on MMP regulation, we initially incubated the B16F10 pooled cells (B16-control, B16-Src, B16-Tks5 and B16-Src/Tks5) overnight in increasing concentrations of doxycycline $(0-2.5 \mu \mathrm{g} / \mathrm{ml})$. Cell monolayers were then washed with PBS to remove serum and incubated for a further $48 \mathrm{~h}$ in serum-free Opti-MEM ${ }^{\circledR}$ medium. The medium was harvested, concentrated and analysed for MMP-2 and MMP-9 activity by gelatin zymography (Fig. 2B). In addition to zymography, samples of cell lysates were also prepared and analysed by immunoblotting in order to confirm induction of Src and Tks5 and also to examine cellular levels of MMP-2 expression. The technique of gelatin zymography identifies gelatinolytic

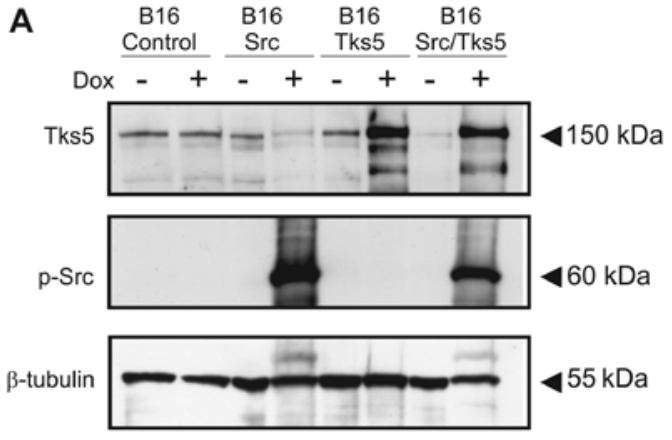

B
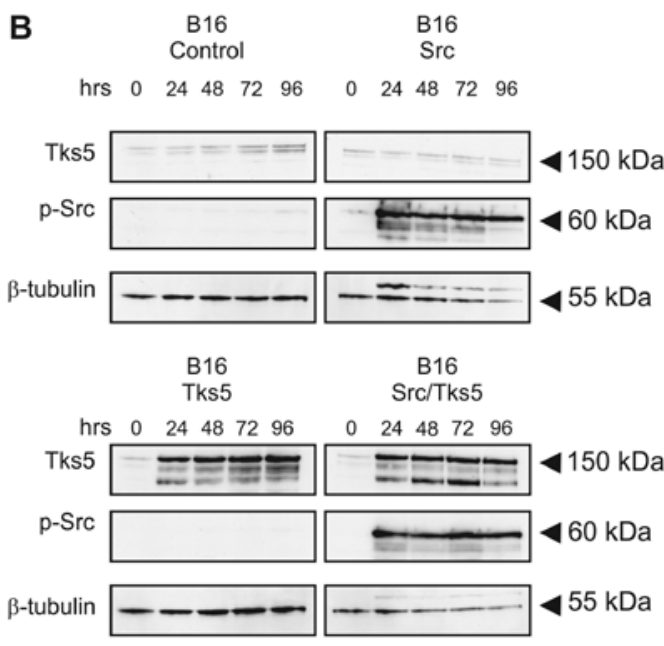

Figure 1. B16F10 inducible pooled cells analysed for Tks5 expression. (A) Cells were incubated with $2.5 \mu \mathrm{g} / \mathrm{ml}$ doxycycline hyclate overnight prior to the preparation of whole cell lysate. Total protein $(20 \mu \mathrm{g})$ was analysed on a NuPAGE Novex 4-12\% Bis-Tris gel. (B) Maintenance of Tks5 expression in B16F10 inducible pooled cells by doxycycline over 4 days. Doxycycline $(2.5 \mu \mathrm{g} / \mathrm{ml})$ was added to the cells at time $=0$. Samples of whole cell lysate were prepared at the indicated times. Total protein $(20 \mu \mathrm{g})$ was subjected to immunoblot analysis with the indicated antibodies.

activity in biological samples electrophoresed under nonreducing conditions in SDS gels impregnated with gelatin. It is a useful tool for the detection of the MMP-2 and MMP-9 gelatinases. Removal of the SDS from the gel in a Triton X-100 buffer promotes renaturation of the MMP enzymes, which in turn can degrade the gelatin leaving a cleared zone which can be detected after Coomassie staining of the undigested gelatin in the gel.

The predominant secreted MMP in the B16F10 cells was found to be MMP-2 (Fig. 2B). The molecular weight of the inactive and mature forms of the MMP-2 zymogen is 68 and $62 \mathrm{kDa}$ respectively $(35,36)$. The levels of the mature form of MMP-2 were enhanced when B16-Src, B16-Tks5 and B16-Src/Tks5 cells were treated with doxycycline, but most prominently in the B16-Src/Tks5 cells. Densitometric analysis of MMP activity is shown in Fig. 2C. A significant difference $(\mathrm{p}<0.01)$ in the activated or mature form of MMP-2 was evident in the B16-Tks5/Src cells compared to the B16-control, Src and Tks5 cells, suggesting increased activation of MMP-2 when Tks5 and Src are overexpressed. A significant increase $(\mathrm{p}<0.05)$ in pro-MMP-2 secretion was observed primarily in the Src overexpressing cells. MMP-9 activity $(92 \mathrm{kDa})$ was also detectable but appeared to be at 

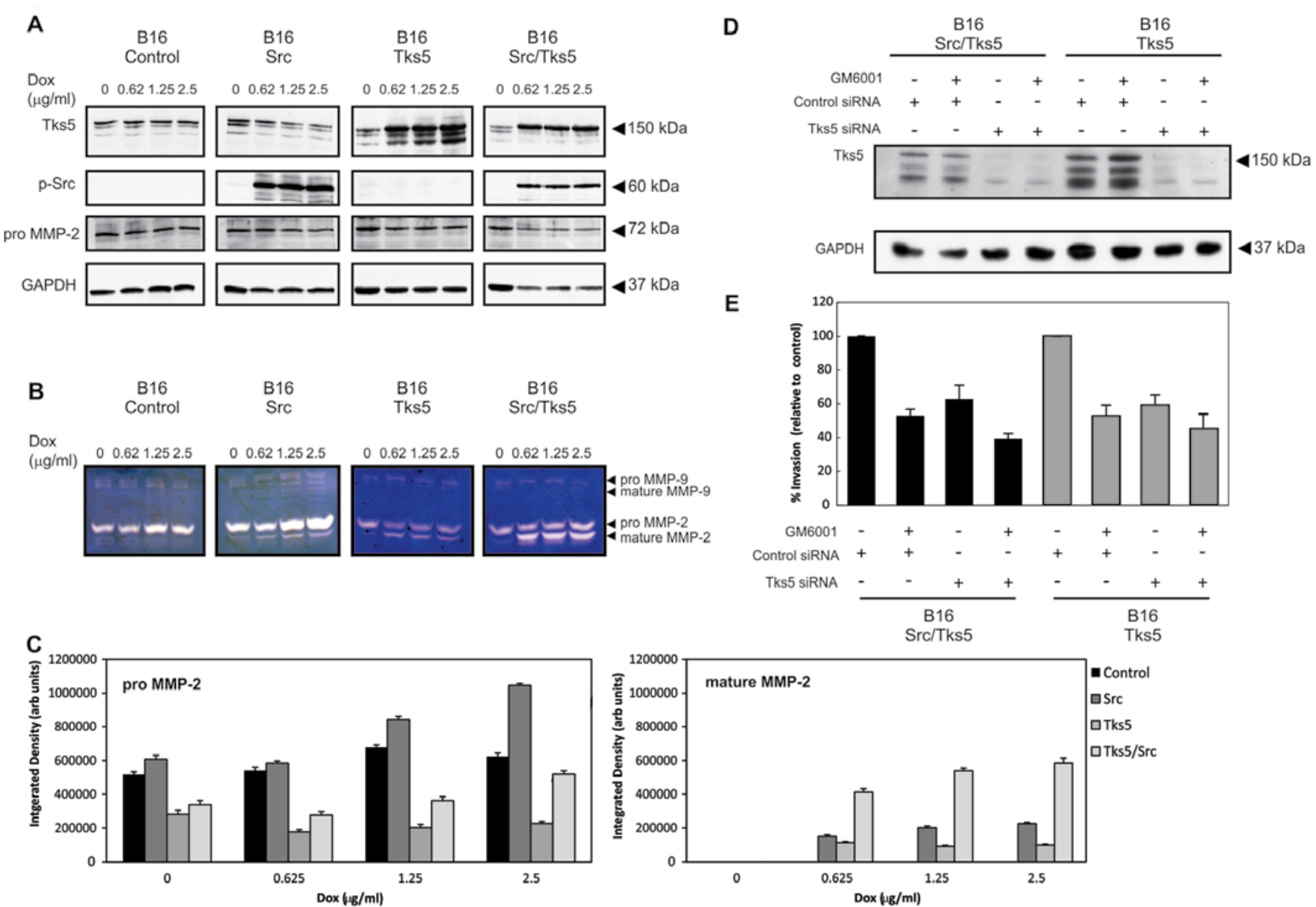

E
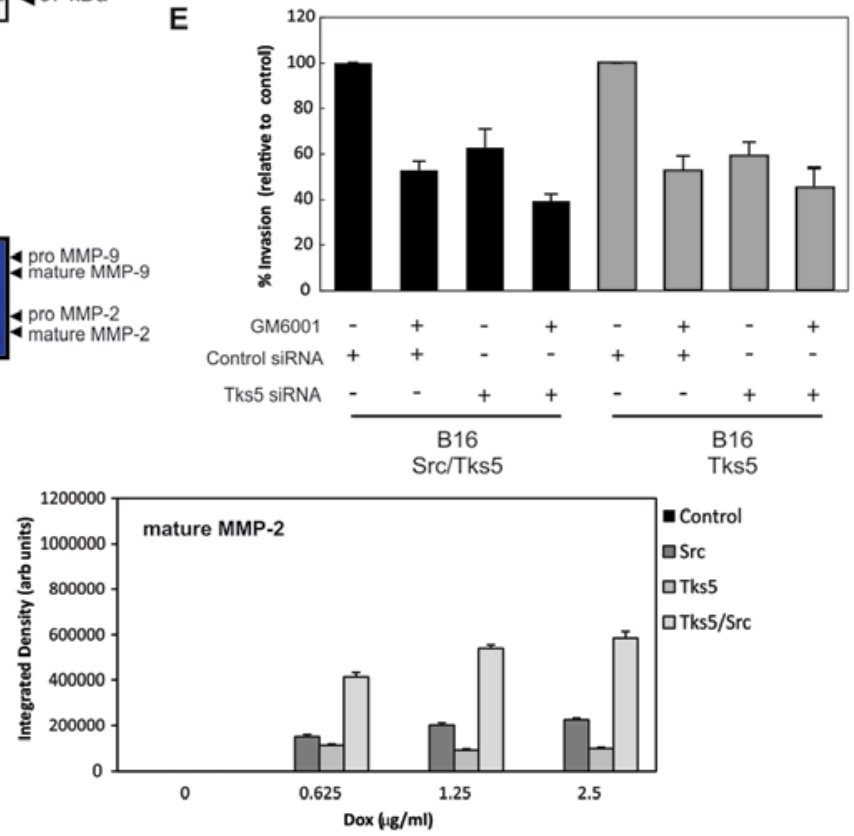

Figure 2. Induction of Src/Tks5 overexpression in B16F10 pooled cells enhances MMP-2 secretion and activation. The B16F10 doxycycline inducible pooled clones seeded in $10-\mathrm{cm}$ plates were induced with $0-2.5 \mu \mathrm{g} / \mathrm{ml}$ doxycycline overnight and then the cells were washed with PBS and incubated in serum-free OptiMEM medium for $48 \mathrm{~h}$. (A) Total protein $(20 \mu \mathrm{g})$ was analysed on a NuPAGE Novex 4-12\% Bis-Tris gel and probed with Tks5, p-Src, MMP-2 (H76) and GAPDH antibodies. (B) Concentrated conditioned medium (12 $\mu \mathrm{l})$ obtained from $10 \mathrm{ml}$ of medium was analysed by electrophoresis with a Novex 10\% zymogram (gelatin) gel. The gel was stained with Coomassie blue to reveal the white bands which correspond to in-gel proteolysis by immature and processed forms of MMP-9 and MMP-2. Inactive zymogen is referred to as pro-MMP-9 or pro-MMP-2 and the mature proteases are generated by proteolytic cleavage for maturation. Tks5 is required for protease-dependent invasion. B16F10 doxycycline induced Tks5 and Tks5/Src pooled cells were transfected with control (Ctrl) or Tks5 siRNA $(100 \mathrm{nM})$ prior to being seeded on the BD BioCoat Matrigel invasion assay and incubated with $10 \mu \mathrm{M}$ GM6001 (or vehicle alone) to monitor the protease dependence of invasion through the Matrigel ${ }^{\circledR}$. (C) The integrated intensity of the pro and mature MMP-2 bands was determined in zymograms such as the representative one presented in Fig. 2B using ImageJ. Densitometric analysis showed a significant increase $(p<0.01)$ in mature MMP-2 for the Tks5/Src-overexpressing cells. Statistically significant ( $\mathrm{p}<0.05$, Student's t-test) secretion of the inactive (pro) MMP-2 form was observed in the Srcoverexpressing cells $(0.625,1.25$ and $2.5 \mu \mathrm{g} / \mathrm{ml}$ Dox $)$ and Src/Tks5-overexpressing cells (1.25 and $2.5 \mu \mathrm{g} / \mathrm{ml}$ Dox). B16F10 Src/Tks5 and Src-overexpressing cells were subjected to Tks5 siRNA-mediated knockdown or treatment with the MMP inhibitor, GM6001 to determine if Tks5-mediated invasion in these cells was protease dependent. The invasive capacity of the cells was examined with a Matrigel invasion assay (E) and Tks5 expression levels verified by western blotting (D). The invasion of each listed experimental group is expressed as a percentage of the control siRNA-treated cells that were not exposed to the GM6001 inhibitor, and $20 \mu \mathrm{g}$ of total protein from whole cell lysate was also analysed on a NuPAGE Novex 4-12\% Bis-Tris gel and probed with Tks5 and GAPDH antibodies (D). The invasive capacity of the Tks5 siRNA- or GM6001-treated cells was significantly reduced ( $<<0.05$, Student's t-test) compared to the control siRNA duplex treated cells (E).

much lower levels compared to MMP-2 in these cells. The overall levels of the inactive MMP-2 zymogen were increased in the culture medium of the B16-Src cells indicating that Src overexpression (Fig. 2A) led to an increase in MMP-2 secretion. In the B16-Src and B16-Tks5 cells, there was an increase in the mature MMP-2 zymogen implying that the induced overexpression of Src or Tks5 promoted MMP-2 processing. Interestingly, the simultaneous overexpression of Src and Tks5 resulted in an apparent additive effect of MMP-2 processing. We previously showed that Tks5 via an Src-Tks5-Nck signalling pathway is important in the formation and activity of invadopodia in B16F10 melanoma cells (24).
Tks5 is required for protease-dependent invasion. The data in Fig. 2A and B suggest that Tks5 overexpression promotes at least in part matrix degradation through the increased secretion and activation of MMP-2. This prompted an assessment of the contribution of matrix metalloproteases to the Tks5-dependent invasion of B16F10 cells through Matrigel. B16-Tks5 and B16-Src/Tks5 pooled cells were treated with the MMP inhibitor, GM6001, and their invasive capacity was compared to untreated cells using a Matrigel invasion system (Fig. 2E). GM6001 is a broad spectrum inhibitor of MMP-1, MMP-2, MMP-3, MMP-7, MMP-8, MMP-9, MMP-12, MMP-14 and MMP-26, with MMP-2 possessing the lowest $\mathrm{IC}_{50}$ value at $0.4 \mathrm{nM}$. The invasive capacity of the 

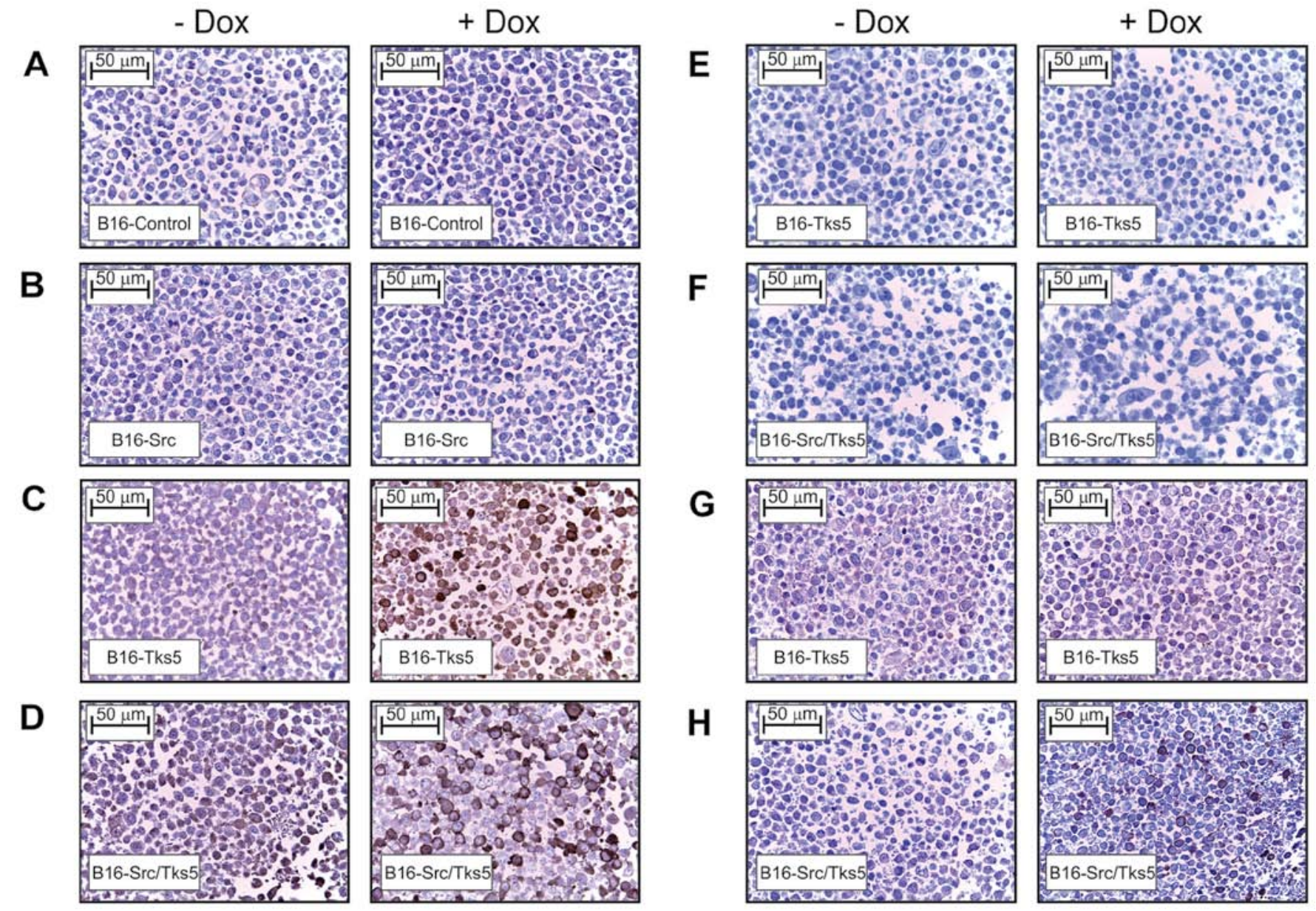

G
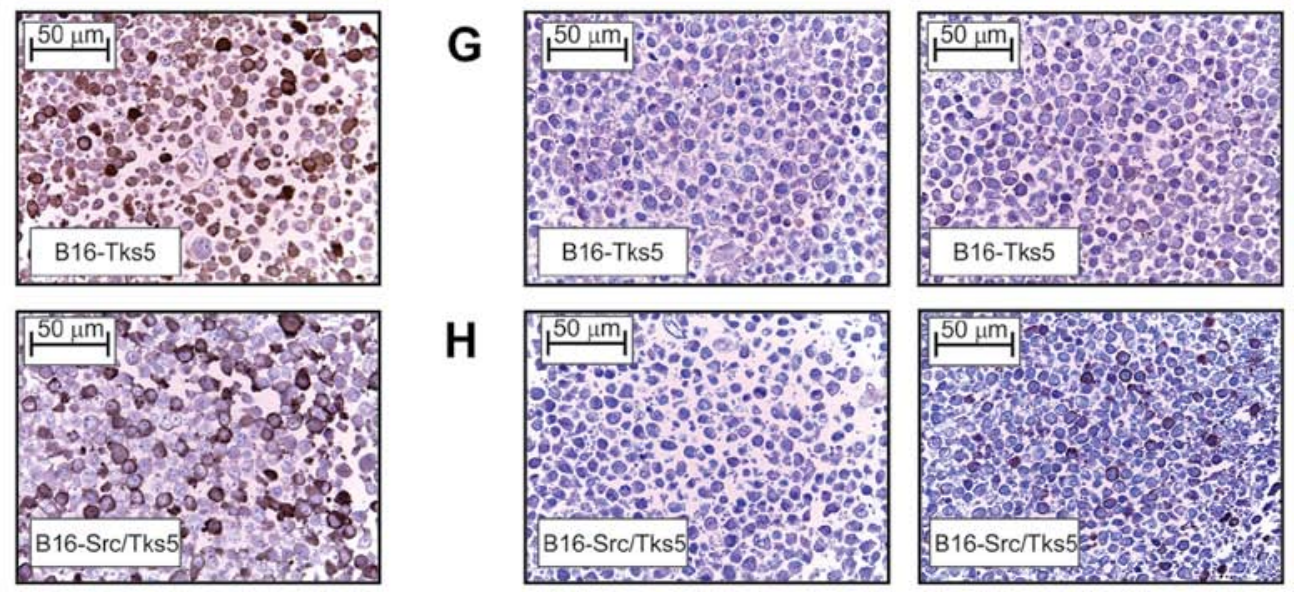

$\mathrm{H}$
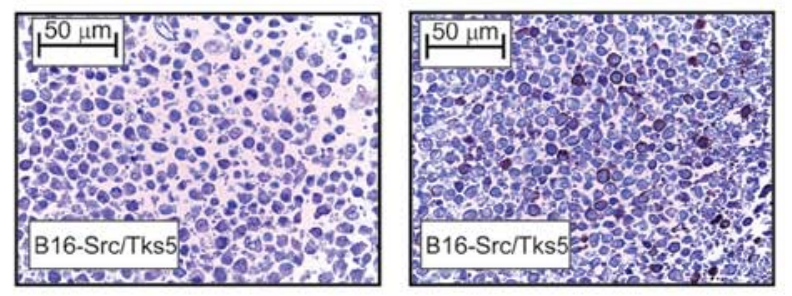

Figure 3. Optimization of Tks5 antigen retrieval using paraffin-embedded pellets of B16F10 pooled inducible cells. (A) B16-control, (B) B16-Src, (C) B16-Tks5 and (D) B16-Src/Tks5 sections were subjected to antigen retrieval with citrate buffer (pH 6.0) and an overnight incubation with the Tks5 antibody (1:100). (E) B16-Tks5 and (F) B16-Src/Tks5 sections were subjected to antigen retrieval with citrate buffer ( $\mathrm{pH}$ 6.0) and overnight incubation in the antibody diluent alone. (G) B16-Tks5 and (H) B16-Src/Tks5 sections were not subjected to antigen retrieval but were incubated overnight with the Tks5 antibody (1:100) alone. After the overnight incubation all sections were incubated with a biotinylated secondary antibody and developed with a DAB peroxidase kit to reveal Tks5 expression (brown) and counterstained with haematoxylin. Brightfield images were captured on a Leica DM750 microscope coupled to a Leica ICC50HD camera with a 10X objective (scale bar, $50 \mu \mathrm{m}$ ). The absence of brown cellular staining indicates the absence of any detectable Tks5 expression.

B16-Tks5 and B16-Src/Tks5 pooled cells transfected with the control siRNA duplex was reduced upon treatment with GM6001. A similar reduction in invasive capacity was also observed for both groups when the cells were transfected with the Tks5 siRNA duplex alone. There was a further reduction in invasion when cells were transfected with the Tks5 siRNA duplex and treated with GM6001 suggesting that Tks5 is required for the proteolytically driven invasion of Matrigel by $\mathrm{B} 16 \mathrm{~F} 10$ cells.

Tks5 is overexpressed in human cancer. To optimize conditions for immunohistochemical detection of Tks5 expression in formalin-fixed paraffin-embedded human tissue samples, 'mock' tissue blocks were prepared from B16F10 pooled cell lines where overexpression of Tks5 could be induced by treatment of cells with doxycycline. To emulate tissue that is formalin-fixed and paraffin-embedded, cells were pelleted, fixed and embedded in paraffin, then subjected to sectioning at $5 \mu \mathrm{m}$ before antigen retrieval with citrate buffer ( $\mathrm{pH}$ 6.0) and probing with the Tks5 antibody. The cell lines that were analysed in this fashion were B16-control, B16-Src, B16-Tks5 and B16-Src/Tks5. Fig. 3A-H shows that the doxycycline-induced expression of Tks5 was clearly detected when antigen retrieval was utilized (C and D) relative to the endogenous Tks5 levels in the B16-control (A and B) and also compared to the overnight incubation of the Tks5 antibody without antigen retrieval (G and H). No staining was observed in Tks5 (E) or Tks5/Src (F) overexpressing cells subjected to antigen retrieval without the Tks5 antibody present in the antibody diluent.

The optimized protocol incorporating antigen retrieval was then used to examine the relative expression of Tks5 in a human cancer tissue microarray containing specimens of breast, colon, lung and prostate tumours. Overall, the array consisted of 56 tissue cores comprised of 16 normal (two different cases for each normal sample and two cores from each case) and 40 tumour unmatched samples (five different cases for each cancer and two cores from each case). According to the manufacturer data sheet supplied with the tissue microarray, tissue identification and diagnosis were performed by certified pathologists and no further staging or clinical information was supplied. The tissue microarray was stained for Tks5 expression and was found to be relatively low in all of the normal tissues analysed as indicated by the results in Fig. 4. Remarkably, by comparison, all tissue cores from 
A
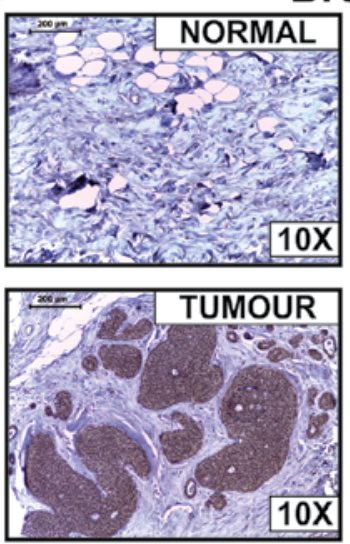

C
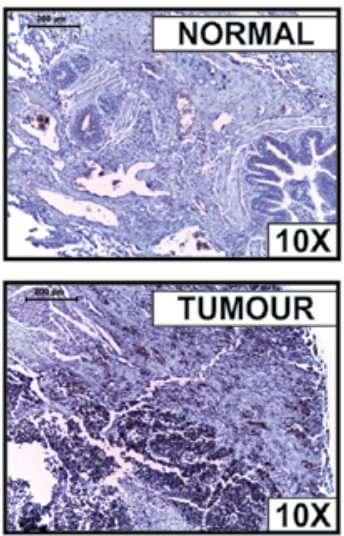

E

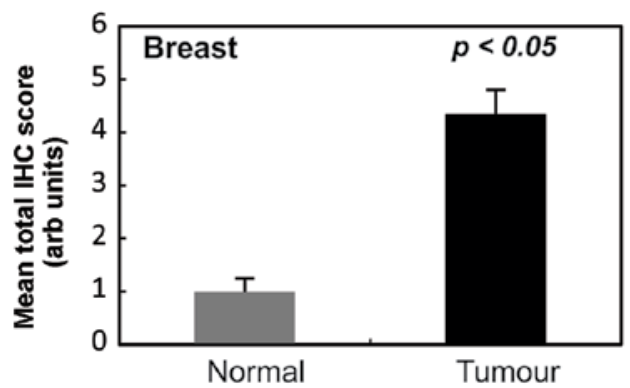

G

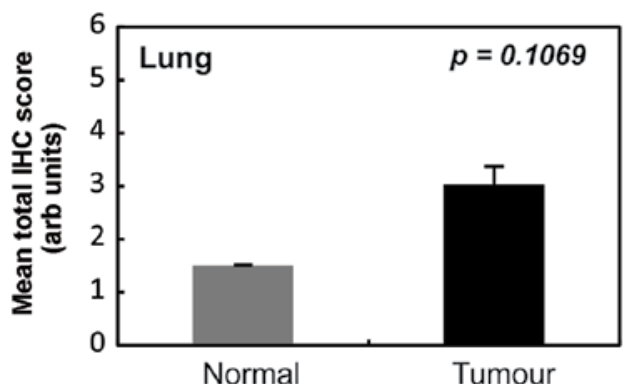

B
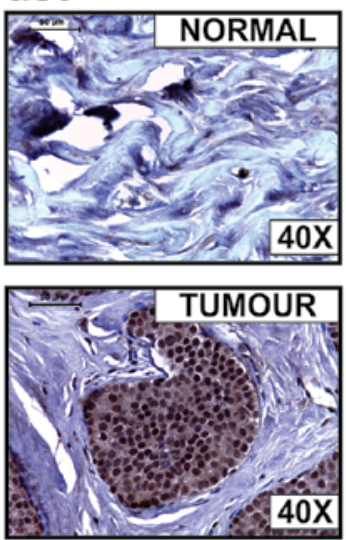

Lung
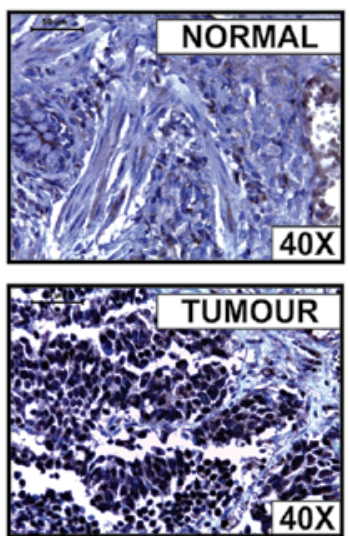

Tumour

Tumour

D

F

H

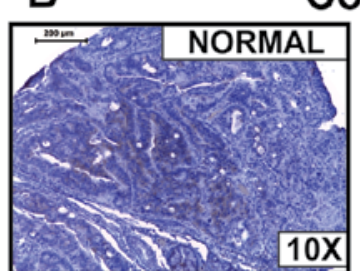

Colon

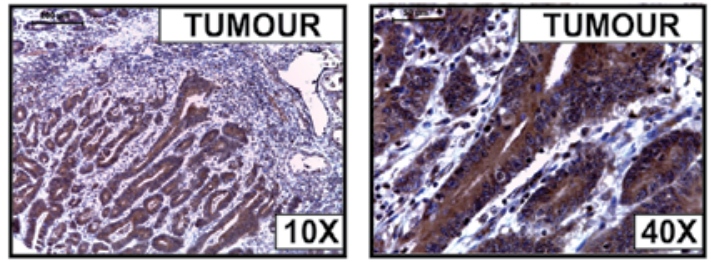

Prostate
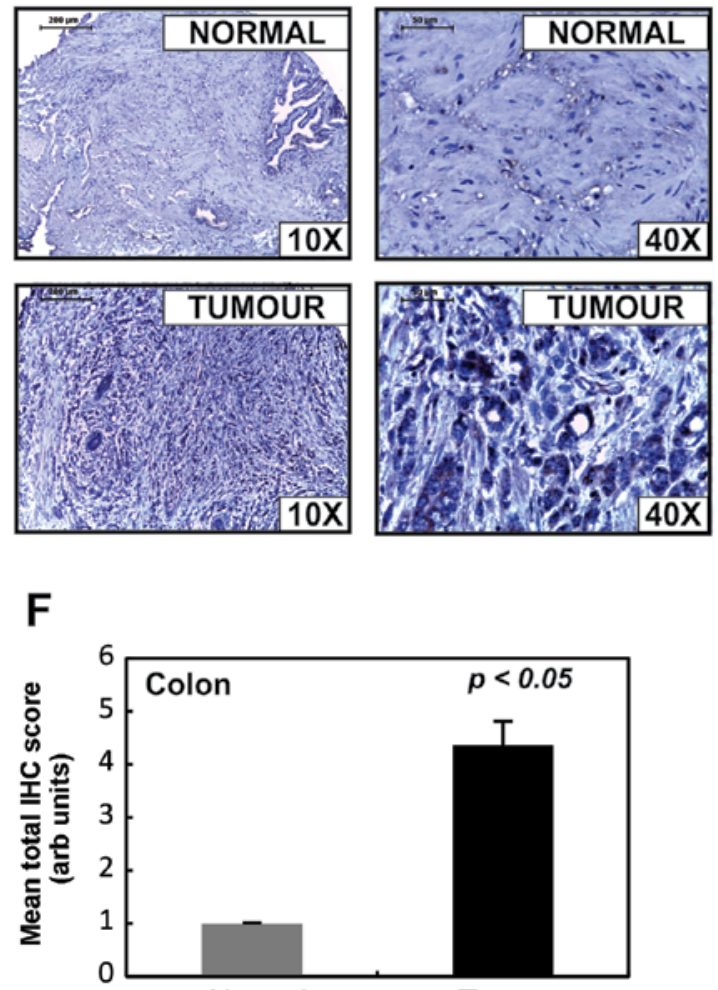

Normal

Tumour

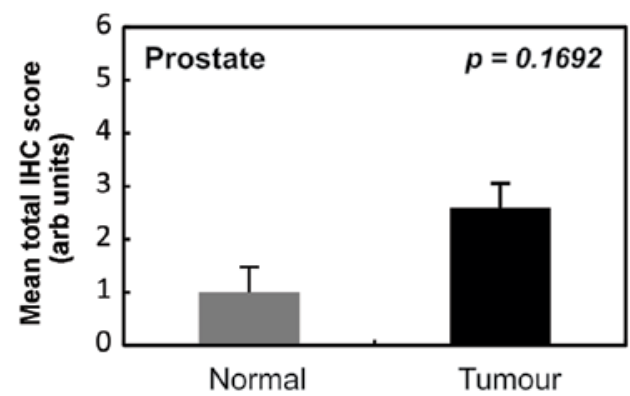

Figure 4. Tks5 is overexpressed in human cancers. Histological sections of paraffin-embedded human cancer tissue microarrays subjected to Tks5 antigen retrieval (citrate buffer, pH 6.0, Tks5 antibody, 1:100) overnight. (A) Breast, (B) colon, (C) lung and (D) prostate tissues include 2.00-mm cores of tumour and corresponding normal tissues (Chemicon Select Tissue Array). Brightfield images were captured with a Leica ICC50HD camera using 10 and 40X objectives (scale bars, 200 and $50 \mu \mathrm{m}$, respectively). Tks5 expression was significantly higher in the breast and colon tumour tissues. The tissue microarray slides were reviewed and scored independently by two observers blinded to the information supplied with the slides and a 'total IHC score' compiled for each core on the tissue microarrays. Total IHC score, tumour cell proportion (score, 1,2 or 3) x staining intensity (score, 1,2 or 3). This is graphically represented in (E) breast, (F) colon, (G) lung and (H) prostate. Error bars indicate SEM. (E) Breast and (F) colon show a significant difference ( $<<0.05$, Student's t-test) in mean total IHC score for Tks5 staining between normal and tumour tissues. 


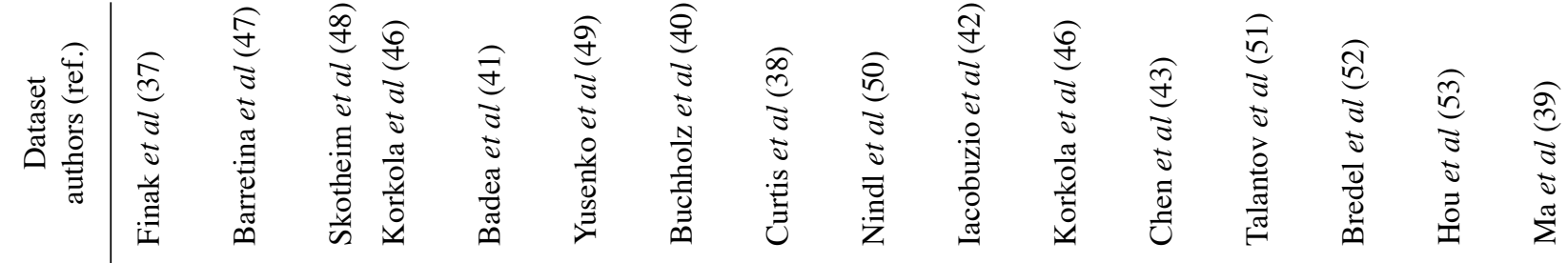

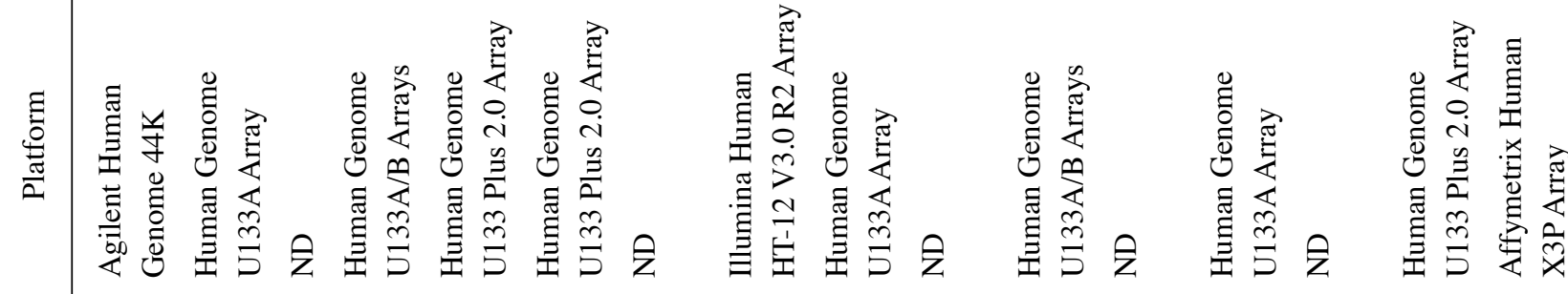

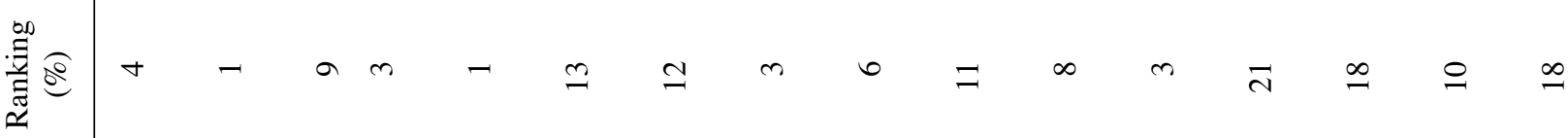

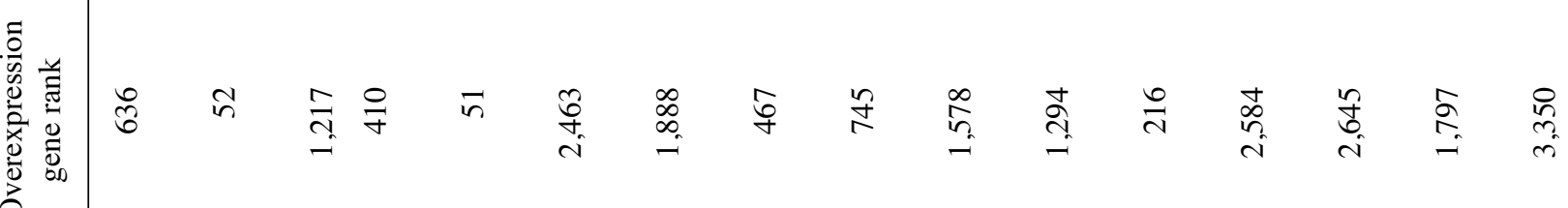
ठे

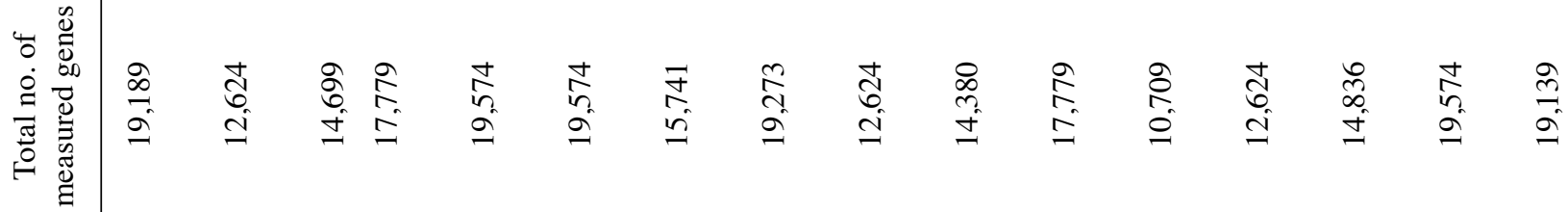

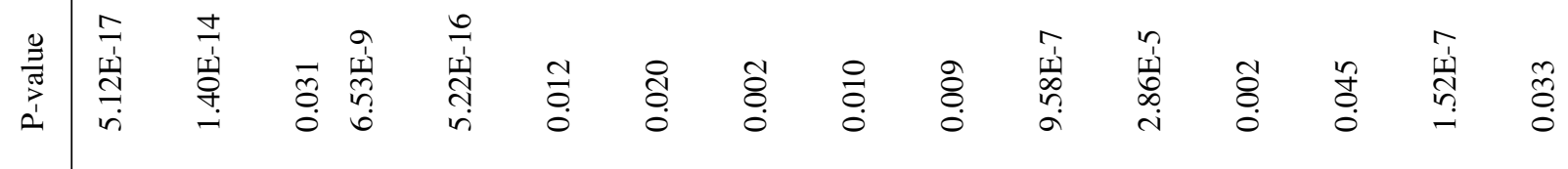

离

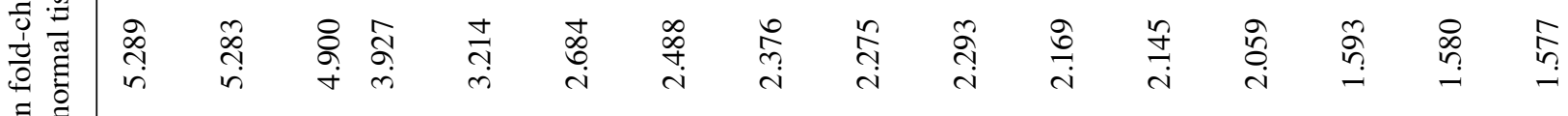

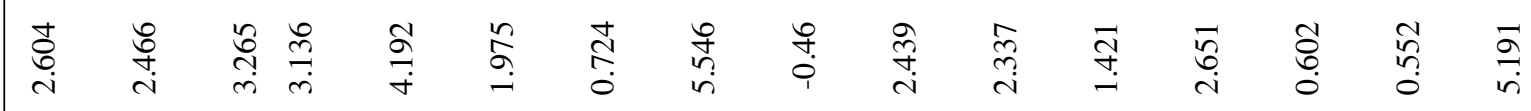

莺

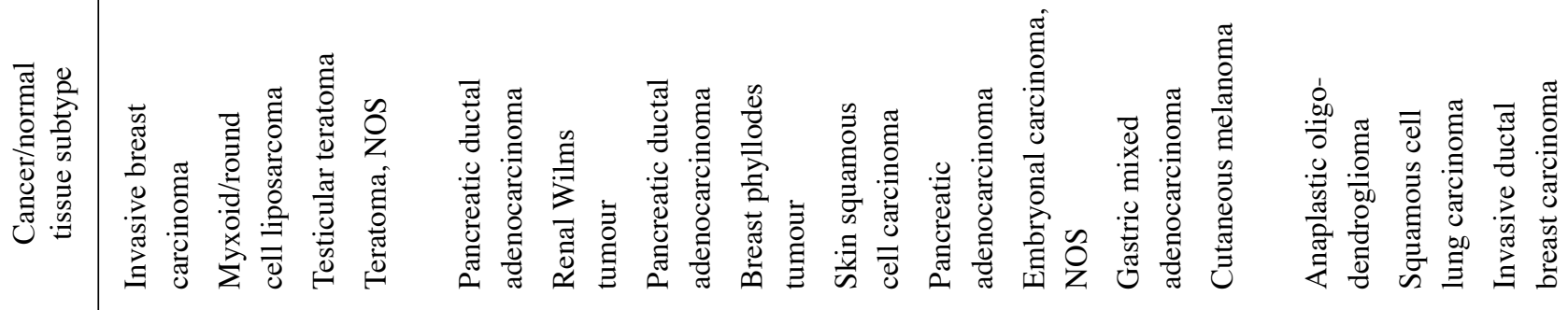


tumour samples showed increased Tks5 expression albeit to varying degrees as assessed independently and enumerated in the 'mean total IHC score' (Fig. 4E-H).

Data mining on the Oncomine database. We systematically compared Tks5 mRNA gene expression levels across a number of human cancers and the corresponding normal tissues using datasets from the Oncomine database (Table I). Overexpression of Tks5 in tumour tissue compared to normal tissue was found in three independent breast cancer studies $(\mathrm{p}=5.12 \mathrm{E}-17, \mathrm{p}=0.002$ and $\mathrm{p}=0.033)(37-39)$; in pancreatic adenocarcinoma compared to normal pancreas in three independent studies ( $\mathrm{p}=5.22 \mathrm{E}-16, \mathrm{p}=0.020$ and $\mathrm{p}=0.009)(40-42)$; in gastric adenocarcinoma compared to the normal tissue counterpart in three independent studies $(\mathrm{p}=2.86 \mathrm{E}-5, \mathrm{p}=1.80 \mathrm{E}-4$ and $\mathrm{p}=0.020)(43-45)$ and a study comparing seminoma to normal testis ( $\mathrm{p}=6.53 \mathrm{E}-9$ and $\mathrm{p}=9.58 \mathrm{E}-7)(46)$. Overexpression of Tks5 was also found in sarcoma $(\mathrm{p}=1.40 \mathrm{E}-14)(47)$, testicular teratoma $(\mathrm{p}=0.031)(48)$, renal $(\mathrm{p}=0.012)(49)$, skin squamous cell carcinoma $(\mathrm{p}=0.010)(50)$, cutaneous melanoma $(\mathrm{p}=0.002)(51)$, anaplastic oligodendroglioma $(\mathrm{p}=0.045)(52)$, squamous cell lung carcinoma $(\mathrm{p}=1.52 \mathrm{E}-7)$ (53) and colon $(\mathrm{p}=2.13 \mathrm{E}-6)$ (54) cancers. P-values were determined by Student's t-test and those $<0.05$ were considered to indicate a statistically significant result.

Thirteen independent studies also showed an association between Tks5 overexpression and poorer prognosis at 1-, 3- or 5-year survival post-diagnosis in various types of cancers (Table II). It is also interesting to note that the Tks5 overexpression gene rank for all the datasets was in the top $15 \%$, with the majority falling within the top $10 \%$ of all overexpressed genes affecting patient prognosis within their respective studies. Two studies (squamous cell carcinoma of the oesophagus (55) and gastric diffuse adenocarcinoma (43) which had a follow-up from 1 to 3 years post-diagnosis showed an increase in the Tks5 gene expression with progression from 1 to 3 years post-diagnosis. Given that Tks5 appeared to be of prognostic significance in a number of cancers, we also investigated whether there was a difference in Tks5 expression between primary and metastatic cancer tissue. Intriguingly, an increase in Tks5 expression was observed in metastatic tissue compared to primary cancer tissue in four studies; ovarian (56,57), breast (58) and prostate (59) (Fig. 5). We also examined the occurrence of metastatic events post-diagnosis being linked to Tks5 expression. Six studies (60-64) covering the time frame of 1,3 or 5 years post-diagnosis showed that there was an increase in Tks5 expression in the patients that had recorded a metastatic event compared to the ones that had none (Fig. 6). This data suggest that Tks5 overexpression is linked to an increase in metastatic potential which may also be associated with a poorer prognostic outcome.

\section{Discussion}

The ability of cells to migrate and invade through the surrounding ECM and across tissue boundaries is crucial not only for physiological processes that occur during normal development, wound healing and immune responses, but importantly for the progression of pathological conditions such as arthritis, vascular diseases and tumour cell invasion 
Table II. Tks5 gene expression and clinical outcome.

\begin{tabular}{|c|c|c|c|c|c|c|c|}
\hline Cancer type & $\begin{array}{l}\text { Year of } \\
\text { death }\end{array}$ & $\begin{array}{l}\text { Mean fold-change } \\
\text { vs. normal tissue }\end{array}$ & P-value & $\begin{array}{l}\text { Total measured } \\
\text { genes }\end{array}$ & Gene rank & $\begin{array}{c}\text { Gene rank } \\
(\%)\end{array}$ & $\begin{array}{c}\text { Dataset } \\
\text { authors (ref.) }\end{array}$ \\
\hline Oesophageal squamous & 1 & 1.370 & 0.019 & 19,574 & 1,444 & 8 & Aoyagi et al (55) \\
\hline cell carcinoma & 3 & 1.611 & 0.032 & 19,574 & 1,837 & 10 & Aoyagi et al (55) \\
\hline Ovarian carcinoma & 1 & 1.074 & 0.038 & 12,624 & 630 & 5 & Bonome et al (76) \\
\hline Gastric intestinal type & 1 & 1.307 & 0.051 & 10,709 & 1,370 & 13 & Chen et al (43) \\
\hline adenocarcinoma & 3 & 1.271 & 0.030 & 10,709 & 425 & 4 & Chen et al (43) \\
\hline Gastric diffuse & 1 & 1.245 & 0.056 & 10,709 & 856 & 8 & Chen et al (43) \\
\hline adenocarcinoma & 3 & 1.415 & 0.023 & 10,709 & 451 & 5 & Chen et al (43) \\
\hline Glioblastoma & 1 & 1.347 & 0.025 & 17,779 & 903 & 6 & Freije et al (77) \\
\hline Adrenal cortex carcinoma & 1 & 1.131 & 0.050 & 19,574 & 2,871 & 15 & Giordano et al (78) \\
\hline Squamous cell lung carcinoma & 1 & 1.129 & 0.049 & 19,574 & 1,243 & 7 & Hou et al (53) \\
\hline Colorectal adenocarcinoma & 1 & 1.109 & 0.047 & 19,574 & 2,344 & 12 & Smith et al (79) \\
\hline $\begin{array}{l}\text { Ovarian peritoneal serous } \\
\text { adenocarcinoma }\end{array}$ & 1 & 1.291 & $3.72 \mathrm{E}-4$ & 19,574 & 26 & 1 & Tothill et al (57) \\
\hline Renal papillary renal & 1 & 1.518 & 0.001 & 19,574 & 134 & 1 & Yang et al (49) \\
\hline cell carcinoma & 5 & 1.267 & 0.005 & 19,574 & 459 & 3 & Yang et al (49) \\
\hline $\begin{array}{l}\text { Poorly differentiated } \\
\text { synovial sarcoma }\end{array}$ & 3 & 1.592 & 0.010 & 19,574 & 180 & 1 & Nakayama et al (80) \\
\hline Astrocytoma & 3 & 1.418 & 0.019 & 17,779 & 1,407 & 8 & Phillips et al (81) \\
\hline Squamous cell lung carcinoma & 3 & 1.231 & 0.024 & 20,423 & 904 & 5 & TCGA \\
\hline Medullary breast carcinoma & 5 & 1.561 & 0.048 & 19,273 & 1,065 & 6 & Curtis et al (38) \\
\hline
\end{tabular}

The relationship between Tks5 mRNA expression and clinical outcomes was examined using the Oncomine database. Tks5 overexpression in tumour tissue compared to normal tissue at 1,3 or 5 years post-diagnosis is associated with a poor prognosis. Displayed in this table are the mean fold-changes vs. corresponding normal tissue in each study, overall p-value, overexpression gene rank and percentage ranking for Tks5 in that dataset. Gene expression data are log transformed and normalized as previously described (32).
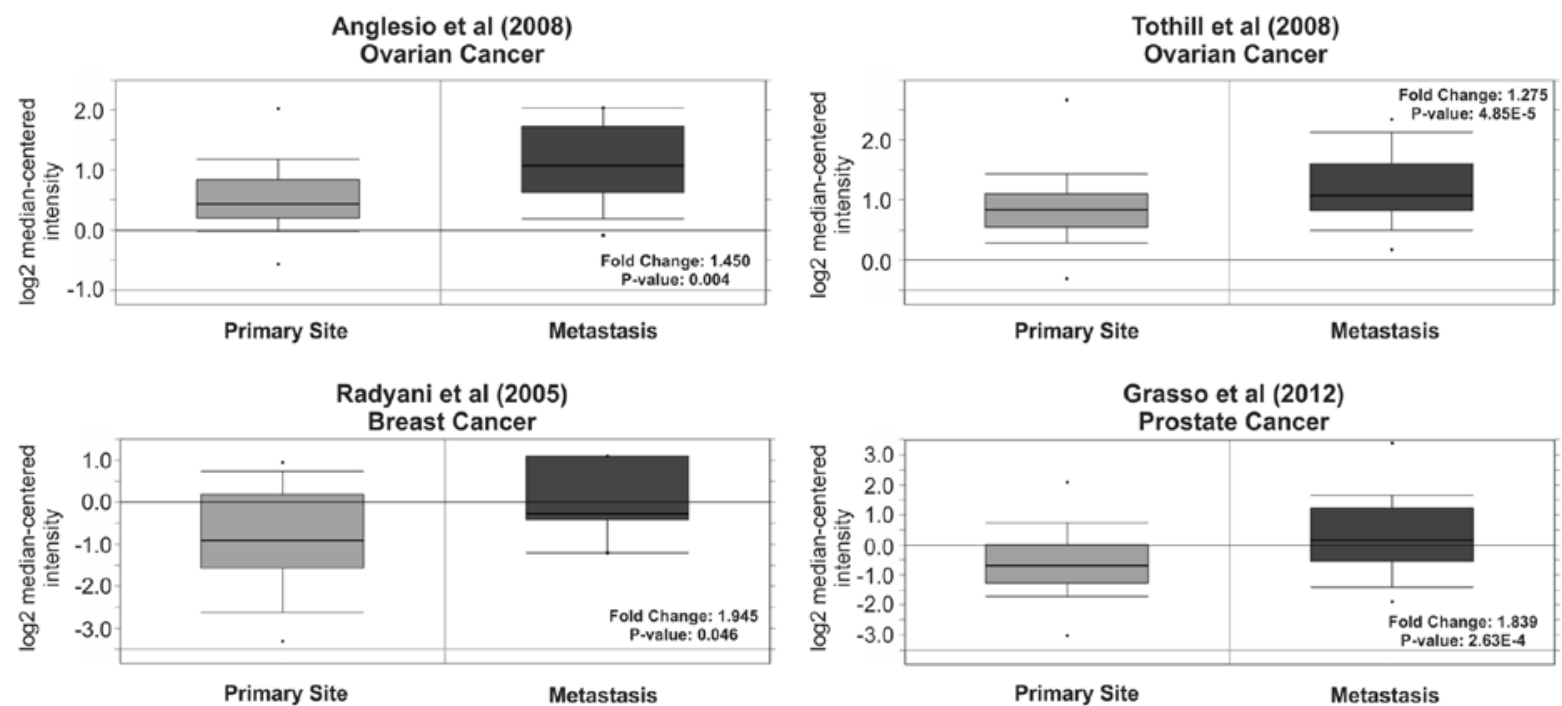

Figure 5. Tks5 is overexpressed in metastatic cancer tissue compared to primary tissue. Expression of Tks5 mRNA was from the respective datasets with primary and metastatic tissue within the Oncomine database. Datasets in a single panel were from the same study. Gene expression data are log transformed and normalized as previously described (32).

and metastasis (65). The cells involved reassemble, reorganize and reform their actin cytoskeleton to facilitate the migration process. The migration of cells also involves the rearrangement of the surrounding ECM which is aided by the secretion 

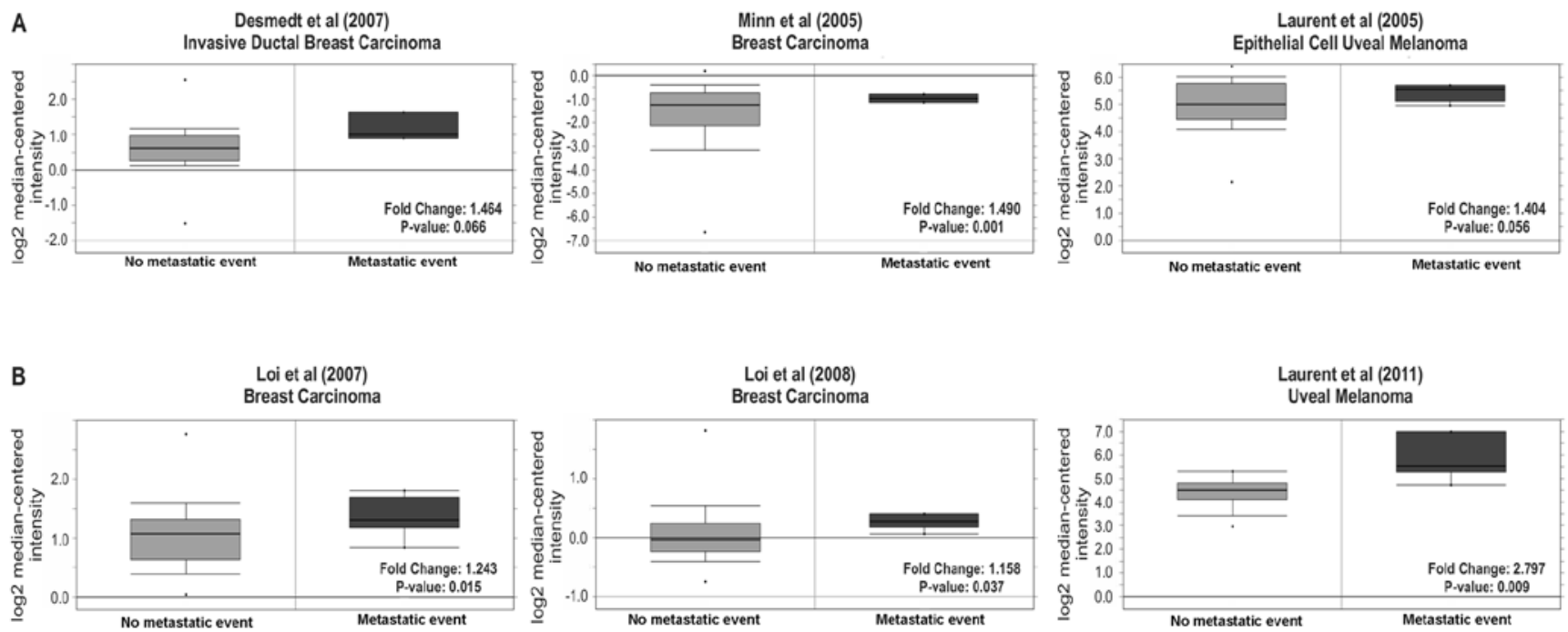

C
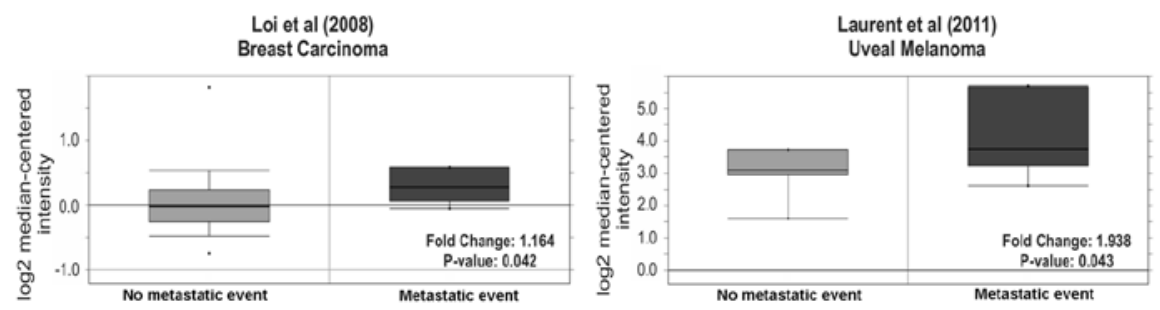

Figure 6. Tks5 is overexpressed in tumour tissue from patients who show evidence of metastatic events. Expression of Tks5 mRNA was extracted from the respective datasets examining metastatic events within the Oncomine database. Datasets from each study are grouped as the time to occurrence of the metastatic events: (A) 1 year, (B) 3 years and (C) 5 years. Gene expression data are log transformed and normalized as previously described (32).

of various proteases arising from actin-based specialized structures known as podosomes in normal cells and invadopodia in tumour cells.

The present study provides evidence that the invadopodiaassociated protein, Tks5, is overexpressed in a number of cancers (breast, colon, lung and prostate cancer). We demonstrated using immunohistochemical staining that Tks5 expression is enhanced in tumour tissues of breast, colon, lung and prostate compared to the normal tissue counterpart and therefore may be of clinical relevance. Our results confirm previously published data also examining Tks5 expression in human cancer (28). Evidence of increased Tks5 expression using immunohistochemical staining in breast carcinoma and invading melanoma cells was observed compared to normal breast and skin tissues. Extensive Tks5 expression was observed in both ductal carcinoma in situ and metastatic breast cancer samples, as well as in the early stage melanoma and metastatic tumour samples.

Even though all cancer tissues in the present study showed increased Tks5 expression, only the breast and colon cancer tissues showed a significant $(\mathrm{p}<0.05)$ increase in Tks5 staining. Having established appropriate conditions for the immunohistochemical detection of Tks5 in tumour tissues, it will be informative in the future to similarly examine the expression of Tks5 in sections of different grades of tumour tissues to determine its distribution in malignant compared to normal surrounding tissues and whether Tks5 levels vary between infiltrating tumour cells at the periphery of all tumours (of different primary origin) and those within the central core region of the tumour itself.

We previously showed that Tks5 via an Src-Tks5-Nck signalling pathway is important in the formation of invadopodia in cancer cells (24). We demonstrated that Src phosphorylates Tks5 at Y557, inducing it to associate directly with the SH3-SH2 domain adaptor proteins Nck1 and Nck2 in invadopodia, promoting matrix degrading activity. It has been postulated that once Nck proteins are recruited to invadopodia, they regulate the actin network via Nck SH3 domain-associated proteins such as N-WASP and the Arp2/3 actin nucleation complex $(66,67)$. Potentially, Tks5-Nck mediated actin assembly could regulate protrusive or vesicular trafficking events in invadopodia within cells at the invasive edge of the tumour. The ECM degradation required for the invasive tumour cells in this region may be linked to the focal recruitment and activation of a number of pericellular proteases including the zinc-regulated metalloproteases (MMP-2, MMP-9 and MT1-MMP) (6,7,15). Demonstrating that Tks5 and Nck co-localize at the invading edge of the tumour within the infiltrating cells would further enhance the importance of Tks5 (and binding partners such as Nck) within invadopodia and the invasion process utilized by tumour cells.

Artym et al (15) observed that another regulator of invadopodium formation, cortactin, was recruited to invadopodia preceding the trafficking of MMPs to future sites of ECM degradation. It has also been suggested that a major role of cortactin in invadopodia is to regulate the localization and 
secretion of MMPs by coupling the dynamic actin assembly to the secretory machinery to enhance ECM degradation $(6,7)$. As we previously observed that increased Tks5 expression results in enhanced matrix degradation (24), we generated a doxycycline inducible system of Tks5 overexpression to precisely ascertain if the increase in ECM degradation in these cells is linked to regulation of the MMP component of invadopodium biogenesis. In the present study, we witnessed an increase in the levels of processed mature MMP-2 in the conditioned medium of the B16F10 doxycycline inducible pooled cells overexpressing Src and Tks5. This indicates that increased Src and Tks5 expression results in enhanced processing and activation of MMP-2. It has also been demonstrated that in EGF-treated cells, Tks5 is phosphorylated within minutes and that this is catalyzed by Src (25). The observed secretion and activation of MMP-2 could be mediated through Src-mediated phosphorylation of Tks5 Y557 allowing for Nck recruitment to Tks5 within invadopodia (24). To further ascertain if Tks5 is required for the proteolytic process of invasion, the B16F10 inducible pooled cells were transiently transfected with Tks5 siRNA. A reduction in Tks5 expression also resulted in a considerable decrease in the invasive capacity of the Tks5 siRNA or GM6001-treated cells compared to the control siRNA duplex cells. The invasive capacity of the control cells or Tks5 siRNA-transfected cells was not completely abolished when treated with GM6001, suggesting the existence of a protease-independent method of invasion not dependent on Tks5 expression (68).

The importance of invadopodia has been emphasized by an increasing number of publications in recent years attempting to clarify the key components and regulatory mechanisms of these structures $(5,6,8,9,11,12,14,15,18,27,33,69)$. These studies have shown that invadopodia in a number of cancer cell lines are involved in the reorganization and degradation of the surrounding ECM. Many of these studies have focused on the actin-binding protein, cortactin, which stabilizes the interaction of Arp2/3 with F-actin in the formation of invadopodia $(6,7,15,70-72)$. However, Tks5, as a multi-SH3 domain adaptor protein and also an Src substrate, has been shown to localize to these structures (3) and be a vital component required for their formation in Src transformed fibroblasts (28) and melanoma cells (24).

Finally, given the importance of finding prognostic markers for the diagnosis and treatment of cancers, there has been increasing evidence linking invadopodium-associated proteins, such as cortactin to poor disease-specific survival in patients (73-75). We previously conducted a retrospective pilot study evaluating the possible role of Tks5 as a prognostic marker in human glioma (30). Even though there was no correlation of Tks5 expression with the grade of glioma, we established that Tks5 expression in glioma can predict reduced survival particularly in lower grade astrocytoma and oligoastrocytoma patients. Our current analysis of Tks5 expression in a number of different human cancers in a commercial tissue microarray also showed increased Tks5 expression in other tumour tissue types (breast, colon, lung and prostate cancer) relative to the corresponding normal tissue. These results also support the previous observations (28) and strengthen the rationale for further examining the role of Tks 5 in cancer given the overexpression observed in a number of human cancers.
Our Oncomine data further highlights the need for expanded studies investigating the clinical significance of Tks5 in a range of cancers. The data extracted from the Oncomine compendium showed that enhanced Tks5 expression was higher in metastatic tumours compared to the primary tumours for ovarian, breast and prostate cancer. In addition, there was also a significant increase in the number of metastatic events when Tks5 was overexpressed.

We intend to undertake further studies examining Tks5 expression in tumour tissues of different types of cancers and histological grades and integrating this with the clinical information (including response to therapies) to obtain a more detailed Tks5 prognostic profile, as the commercial tissue array utilized in this study, did not have associated relevant patient survival data linked to the pathological diagnosis. Patients with a poorer prognosis may harbour tumours with a more invasive margin that contain Tks5-positive cells infiltrating further into the surrounding tissue. These cells may also show co-localization of Tks5 binding partners such as Nck, supporting an Src-Tks5-Nck signalling pathway (24). This approach is reinforced by the results from our present analysis of the Oncomine database, where Tks5 overexpression in human types of cancers was listed in the top $10-15 \%$ of all genes that were analysed in the respective cancer datasets. In conclusion, the present study identified Tks5 as a potential prognostic indicator in the range of cancers examined. Secondly, it raises the possibility that Tks5 should be further investigated as a pertinent molecular target in the development of future anticancer agents for personalized therapy of patients harbouring Tks5 overexpressing tumours.

\section{Acknowledgements}

We are grateful to the contributors of data to Oncomine and those who have made their data publicly available.

\section{References}

1. Sporn MB: The war on cancer. Lancet 347: 1377-1381, 1996.

2. Hanahan D and Weinberg RA: Hallmarks of cancer: the next generation. Cell 144: 646-674, 2011.

3. Abram CL, Seals DF, Pass I, et al: The adaptor protein fish associates with members of the ADAMs family and localizes to podosomes of Src-transformed cells. J Biol Chem 278: 16844-16851, 2003.

4. Bowden ET, Barth M, Thomas D, Glazer RI and Mueller SC: An invasion-related complex of cortactin, paxillin and $\mathrm{PKC} \mu$ associates with invadopodia at sites of extracellular matrix degradation. Oncogene 18: 4440-4449, 1999.

5. Buccione R, Caldieri G and Ayala I: Invadopodia: specialized tumor cell structures for the focal degradation of the extracellular matrix. Cancer Metastasis Rev 28: 137-149, 2009.

6. Clark ES and Weaver AM: A new role for cortactin in invadopodia: regulation of protease secretion. Eur J Cell Biol 87: 581-590, 2008.

7. Clark ES, Whigham AS, Yarbrough WG and Weaver AM: Cortactin is an essential regulator of matrix metalloproteinase secretion and extracellular matrix degradation in invadopodia. Cancer Res 67: 4227-4235, 2007

8. Gimona M, Buccione R, Courtneidge SA and Linder S: Assembly and biological role of podosomes and invadopodia. Curr Opin Cell Biol 20: 235-241, 2008.

9. Linder S: The matrix corroded: podosomes and invadopodia in extracellular matrix degradation. Trends Cell Biol 17: 107-117, 2007.

10. Linder S and Aepfelbacher M: Podosomes: adhesion hot-spots of invasive cells. Trends Cell Biol 13: 376-385, 2003. 
11. Weaver AM: Invadopodia: specialized cell structures for cancer invasion. Clin Exp Metastasis 23: 97-105, 2006.

12. Weaver AM: Cortactin in tumor invasiveness. Cancer Lett 265 $157-166,2008$

13. Webb BA, Jia L, Eves R and Mak AS: Dissecting the functional domain requirements of cortactin in invadopodia formation. Eur J Cell Biol 86: 189-206, 2007.

14. Yamaguchi H, Pixley F and Condeelis J: Invadopodia and podosomes in tumor invasion. Eur J Cell Biol 85: 213-218, 2006.

15. Artym VV, Zhang Y, Seillier-Moiseiwitsch F, Yamada KM and Mueller SC: Dynamic interactions of cortactin and membrane type 1 matrix metalloproteinase at invadopodia: defining the stages of invadopodia formation and function. Cancer Res 66 3034-3043, 2006.

16. Daly RJ: Cortactin signalling and dynamic actin networks Biochem J 382: 13-25, 2004

17. Li Y, Tondravi M, Liu J, et al: Cortactin potentiates bone metastasis of breast cancer cells. Cancer Res 61: 6906-6911, 2001.

18. Yamaguchi H, Lorenz M, Kempiak S, et al: Molecular mechanisms of invadopodium formation: the role of the N-WASP-Arp2/3 complex pathway and cofilin. J Cell Biol 168: 441-452, 2005.

19. Staub E, Groene J, Heinze M, et al: An expression module of WIPF1-coexpressed genes identifies patients with favorable prognosis in three tumor types. J Mol Med 87: 633-644, 2009.

20. Hanahan D and Weinberg RA: The hallmarks of cancer. Cell 100: $57-70,2000$.

21. Pao W, Miller V,Zakowski M, et al: EGF receptor gene mutations are common in lung cancers from 'never smokers' and are associated with sensitivity of tumors to gefitinib and erlotinib. Proc Natl Acad Sci USA 101: 13306-13311, 2004.

22. Zeineldin R, Muller CY, Stack MS and Hudson LG: Targeting the EGF receptor for ovarian cancer therapy. J Oncol 2010 : 414676, 2010.

23. Lock P, Abram CL, Gibson T and Courtneidge SA: A new method for isolating tyrosine kinase substrates used to identify fish, an SH3 and PX domain-containing protein, and Src substrate. The EMBO J 17: 4346-4357, 1998.

24. Stylli SS, Stacey TT, Verhagen AM, et al: Nck adaptor proteins link Tks5 to invadopodia actin regulation and ECM degradation. J Cell Sci 122: 2727-2740, 2009.

25. Fekete A, Bőgel G, Pesti S, Péterfi Z, Geiszt M and Buday L: EGF regulates tyrosine phosphorylation and membrane-translocation of the scaffold protein Tks5. J Mol Signal 8: 8, 2013.

26. Balzer EM, Whipple RA, Thompson K, et al: c-Src differentially regulates the functions of microtentacles and invadopodia. Oncogene 29: 6402-6408, 2010.

27. Oikawa $\mathrm{T}$, Itoh $\mathrm{T}$ and Takenawa $\mathrm{T}$ : Sequential signals toward podosome formation in NIH-src cells. J Cell Biol 182: 157-169, 2008

28. Seals DF, Azucena EF Jr, Pass I, et al: The adaptor protein Tks5/Fish is required for podosome formation and function, and for the protease-driven invasion of cancer cells. Cancer Cell 7: $155-165,2005$

29. Murphy DA, Diaz B, Bromann PA, et al: A Src-Tks5 pathway is required for neural crest cell migration during embryonic development. PLoS One 6: e22499, 2011

30. Stylli SS, I ST, Kaye AH and Lock P: Prognostic significance of Tks5 expression in gliomas. J Clin Neurosci 19: 436-442, 2012.

31. Zhu HJ, Iaria J and Sizeland AM: Smad7 differentially regulates transforming growth factor $\beta$-mediated signaling pathways. J Biol Chem 274: 32258-32264, 1999.

32. Rhodes DR, Yu J, Shanker K, et al: ONCOMINE: a cancer microarray database and integrated data-mining platform. Neoplasia 6: 1-6, 2004

33. Ayala I, Baldassarre M, Caldieri G and Buccione R: Invadopodia: a guided tour. Eur J Cell Biol 85: 159-164, 2006.

34. Baldassarre M, Pompeo A, Beznoussenko G, et al: Dynamin participates in focal extracellular matrix degradation by invasive cells. Mol Biol Cell 14: 1074-1084, 2003.

35. Deryugina EI and Quigley JP: Matrix metalloproteinases and tumor metastasis. Cancer Metastasis Rev 25: 9-34, 2006.

36. Deryugina EI, Ratnikov B, Monosov E, et al: MT1-MMP initiates activation of pro-MMP- 2 and integrin $\alpha v \beta 3$ promotes maturation of MMP-2 in breast carcinoma cells. Exp Cell Res 263: 209-223, 2001.

37. Finak G, Bertos N, Pepin F, et al: Stromal gene expression predicts clinical outcome in breast cancer. Nat Med 14: 518-527, 2008 .
38. Curtis C, Shah SP, Chin SF, et al: The genomic and transcriptomic architecture of 2,000 breast tumours reveals novel subgroups. Nature 486: 346-352, 2012

39. Ma XJ, Dahiya S, Richardson E, Erlander M and Sgroi DC: Gene expression profiling of the tumor microenvironment during breast cancer progression. Breast Cancer Res 11: R7, 2009.

40. Buchholz M, Braun M, Heidenblut A, et al: Transcriptome analysis of microdissected pancreatic intraepithelial neoplastic lesions. Oncogene 24: 6626-6636, 2005.

41. Badea L, Herlea V, Dima SO, Dumitrascu T and Popescu I: Combined gene expression analysis of whole-tissue and microdissected pancreatic ductal adenocarcinoma identifies genes specifically overexpressed in tumor epithelia. Hepatogastroenterology 55: 2016-2027, 2008.

42. Iacobuzio-Donahue CA, Maitra A, Olsen M, et al: Exploration of global gene expression patterns in pancreatic adenocarcinoma using cDNA microarrays. Am J Pathol 162: 1151-1162, 2003.

43. Chen X, Leung SY, Yuen ST, et al: Variation in gene expression patterns in human gastric cancers. Mol Biol Cell 14: 3208-3215, 2003.

44. Cho JY, Lim JY, Cheong JH, et al: Gene expression signaturebased prognostic risk score in gastric cancer. Clin Cancer Res 17: 1850-1857, 2011.

45. D'Errico M, de Rinaldis E, Blasi MF, et al: Genome-wide expression profile of sporadic gastric cancers with microsatellite instability. Eur J Cancer 45: 461-469, 2009.

46. Korkola JE, Houldsworth J, Chadalavada RS, et al: Downregulation of stem cell genes, including those in a $200-\mathrm{kb}$ gene cluster at $12 \mathrm{p} 13.31$, is associated with in vivo differentiation of human male germ cell tumors. Cancer Res 66: 820-827, 2006.

47. Barretina J, Taylor BS, Banerii S, et al: Subtype-specific genomic alterations define new targets for soft-tissue sarcoma therapy. Nat Genet 42: 715-721, 2010 .

48. Skotheim RI, Lind GE, Monni O, et al: Differentiation of human embryonal carcinomas in vitro and in vivo reveals expression profiles relevant to normal development. Cancer Res 65 $5588-5598,2005$

49. Yusenko MV, Kuiper RP, Boethe T, Ljungberg B, van Kessel AG and Kovacs G: High-resolution DNA copy number and gene expression analyses distinguish chromophobe renal cell carcinomas and renal oncocytomas. BMC Cancer 9: 152, 2009.

50. Nindl I, Dang C, Forschner T, et al: Identification of differentially expressed genes in cutaneous squamous cell carcinoma by microarray expression profiling. Mol Cancer 5: 30, 2006.

51. Talantov D, Mazumder A, Yu JX, et al: Novel genes associated with malignant melanoma but not benign melanocytic lesions. Clin Cancer Res 11: 7234-7242, 2005.

52. Bredel M, Bredel C, Juric D, et al: Functional network analysis reveals extended gliomagenesis pathway maps and three novel MYC-interacting genes in human gliomas. Cancer Res 65 8679-8689, 2005.

53. Hou J, Aerts J, den Hamer B, et al: Gene expression-based classification of non-small cell lung carcinomas and survival prediction. PLoS One 5: e10312, 2010.

54. Kaiser S, Park YK, Franklin JL, et al: Transcriptional recapitulation and subversion of embryonic colon development by mouse colon tumor models and human colon cancer. Genome Biol 8: R131, 2007.

55. Aoyagi K, Tatsuta T, Nishigaki M, et al: A faithful method for PCR-mediated global mRNA amplification and its integration into microarray analysis on laser-captured cells. Biochem Biophys Res Commun 300: 915-920, 2003.

56. Anglesio MS, Arnold JM, George J, et al: Mutation of ERBB2 provides a novel alternative mechanism for the ubiquitous activation of RAS-MAPK in ovarian serous low malignant potential tumors. Mol Cancer Res 6: 1678-1690, 2008.

57. Tothill RW, Tinker AV, George J, et al: Novel molecular subtypes of serous and endometrioid ovarian cancer linked to clinical outcome. Clin Cancer Res 14: 5198-5208, 2008

58. Radvanyi L, Singh-Sandhu D, Gallichan S, et al: The gene associated with trichorhinophalangeal syndrome in humans is overexpressed in breast cancer. Proc Natl Acad Sci USA 102: 11005-11010, 2005

59. Grasso CS, Wu YM, Robinson DR, et al: The mutational landscape of lethal castration-resistant prostate cancer. Nature 487: 239-243, 2012.

60. Desmedt C, Piette F, Loi S, et al: Strong time dependence of the 76-gene prognostic signature for node-negative breast cancer patients in the TRANSBIG multicenter independent validation series. Clin Cancer Res 13: 3207-3214, 2007. 
61. Minn AJ, Gupta GP, Siegel PM, et al: Genes that mediate breast cancer metastasis to lung. Nature 436: 518-524, 2005.

62. Laurent C, Valet F, Planque N, et al: High PTP4A3 phosphatase expression correlates with metastatic risk in uveal melanoma patients. Cancer Res 71: 666-674, 2011.

63. Loi S, Haibe-Kains B, Desmedt C, et al: Predicting prognosis using molecular profiling in estrogen receptor-positive breast cancer treated with tamoxifen. BMC Genomics 9: 239, 2008.

64. Loi S, Haibe-Kains B, Desmedt C, et al: Definition of clinically distinct molecular subtypes in estrogen receptor-positive breast carcinomas through genomic grade. J Clin Oncol 25: 1239-1246, 2007.

65. Lambrechts A, Van Troys M and Ampe C: The actin cytoskeleton in normal and pathological cell motility. Int J Biochem Cell Biol 36: 1890-1909, 2004.

66. Rohatgi R, Nollau P, Ho HY, Kirschner MW and Mayer BJ: Nck and phosphatidylinositol 4,5-bisphosphate synergistically activate actin polymerization through the N-WASP-Arp2/3 pathway. J Biol Chem 276: 26448-26452, 2001.

67. Rivera GM, Briceño CA, Takeshima F, Snapper SB and Mayer BJ: Inducible clustering of membrane-targeted SH3 domains of the adaptor protein Nck triggers localized actin polymerization. Curr Biol 14: 11-22, 2004.

68. Rowe RG and Weiss SJ: Navigating ECM barriers at the invasive front: the cancer cell-stroma interface. Annu Rev Cell Dev Biol 25: 567-595, 2009

69. Bowden ET, Onikoyi E, Slack R, et al: Co-localization of cortactin and phosphotyrosine identifies active invadopodia in human breast cancer cells. Exp Cell Res 312: 1240-1253, 2006.

70. Kinley AW, Weed SA, Weaver AM, et al: Cortactin interacts with WIP in regulating Arp $2 / 3$ activation and membrane protrusion. Curr Biol 13: 384-393, 2003.

71. Oser M, Yamaguchi $\mathrm{H}$, Mader CC, et al: Cortactin regulates cofilin and N-WASp activities to control the stages of invadopodium assembly and maturation. J Cell Biol 186: 571-587, 2009.
72. Webb BA, Zhou S, Eves R, Shen L, Jia L and Mak AS: Phosphorylation of cortactin by p21-activated kinase. Arch Biochem Biophys 456: 183-193, 2006.

73. Gibcus JH, Mastik MF, Menkema L, et al: Cortactin expression predicts poor survival in laryngeal carcinoma. Br J Cancer 98: 950-955, 2008.

74. Hofman P, Butori C, Havet K, et al: Prognostic significance of cortactin levels in head and neck squamous cell carcinoma: comparison with epidermal growth factor receptor status. Br J Cancer 98: 956-964, 2008.

75. Sheen-Chen SM, Huang CY, Liu YY, Huang CC and Tang RP: Cortactin in breast cancer: analysis with tissue microarray. Anticancer Res 31: 293-297, 2011.

76. Bonome T, Levine DA, Shih J, et al: A gene signature predicting for survival in suboptimally debulked patients with ovarian cancer. Cancer Res 68: 5478-5486, 2008.

77. Freije WA, Castro-Vargas FE, Fang Z, et al: Gene expression profiling of gliomas strongly predicts survival. Cancer Res 64: 6503-6510, 2004

78. Giordano TJ, Kuick R, Else T, et al: Molecular classification and prognostication of adrenocortical tumors by transcriptome profiling. Clin Cancer Res 15: 668-676, 2009.

79. Smith JJ, Deane NG, Wu F, et al: Experimentally derived metastasis gene expression profile predicts recurrence and death in patients with colon cancer. Gastroenterology 138: 958-968, 2010.

80. Nakayama R, Mitani S, Nakagawa T, et al: Gene expression profiling of synovial sarcoma: distinct signature of poorly differentiated type. Am J Surg Pathol 34: 1599-1607, 2010.

81. Phillips HS, Kharbanda S, Chen R, et al: Molecular subclasses of high-grade glioma predict prognosis, delineate a pattern of disease progression, and resemble stages in neurogenesis. Cancer Cell 9: 157-173, 2006. 